\title{
Knowledge and Attitude of Tuberculosis Patients and Their Family Caregivers about Tuberculosis in Minia Governorate
}

\author{
Abdelwaha , A., A; Sadek , R., R; Kotb , S., A \& Ahmed , M., A. \\ Public Health and Community Medicine., Faculty of Medicine., Minia University \\ Community Health Nursing ., Faculty of Nursing., Assiut University \\ Community Health Nursing ., Faculty of Nursing., Beni-suif University
}

\begin{abstract}
:
Background: Tuberculosis continues to pose serious public health problem threats, especially in developing countries. It is a common and often deadly infectious disease caused by mycobacteria, usually Mycobacterium tuberculosis in humans. Tuberculosis usually attacks the lungs but can also affect other parts of the body. Each year, approximately 2 million persons worldwide die of tuberculosis and 9 million become infected. Aim of this study: to assess knowledge and attitude of TB patients and their family caregivers about TB in Minia governorate. Subject and Methods: Cross sectional research design was utilized in the present study. This study was carried out in Minia governorate; three districts namely Beni-mazar, Minia and Mallawy that were selected randomly. The study sample included 125 patients' and 173 family caregivers. Two tools were used in this study. First tool: consists of three parts: Part I; included sociodemographic characteristics , Part II; included environmental assessment sheet. Part III; included Knowledge of patient and their families about Tuberculosis disease. Second tool: Likert scale used to assess attitude of patients and their families about tuberculosis. Results: This study illustrated that the age of TB patients and their family caregivers ranged between (18-60) years with the mean age $(34.8 \pm 13.7,38.8 \pm 13.9)$, respectively. Concerning the educational level, it was found that the highest percentage in both groups were illiterate, it represents $43.2 \%$ in the TB patients and $43.9 \%$ in the family caregivers, respectively. Also, the present study showed that the majority of TB patient and their families had poor knowledge and negative attitude about TB. Conclusion: the findings of this study illustrated that more than half of patient and their families had poor knowledge about TB and more than three quarters of them had negative attitude especially in rural areas. Recommendation: there is a strong need for increasing patients and family caregiver's awareness about TB transmission and prevention.
\end{abstract}

\section{Key Words: Tuberculosis, prevention and family}

\section{Introduction:}

Tuberculosis (TB) is "an airborne infectious disease caused by various strains of mycobacteria, usually Mycobacterium tuberculosis (M.TB) in human or Mycobacterium bovis in cattle or other animals. It most commonly affects the lungs, but can infect other parts of the body". It is the most common fatal infectious disease in the world: one-third of the world's population is currently infected and more than 1.5 million people die each year due to tuberculosis. (Konstantinos, 2010; Kyle \& Carman, 2013).

It spreads through the air, when people who have the disease cough, sneeze, or spit; they propel TB germs, known as bacilli, into the air. A person needs only to inhale a small number of these to be infected. They can remain suspended in the air for several hours, depending on the environment. When a person breathes in M. TB, the bacteria can settle in the lungs and begin to grow. From there, they can move through the blood to other parts of the body. TB in the lungs can be infectious because the bacteria are easily spreading to other people. TB in other parts of the body, such as the kidney or spine, is usually not infectious. (Sillah et al, 2007; CDC, 2009b).

Poor air quality and overcrowding within homes as a result of inadequate ventilation and the presence of mold and smoke contribute to poor respiratory health in general and have been implicated in the spread and/or outcome of tuberculosis (TB). (Wanyeki, et al., 2006)

Treatment requires the use of multiple drugs for several months. With appropriate antibiotic treatment, TB can be cured in most people. and usually combines several different antibiotic drugs that are given for at least 6 months, sometimes for as long as 12 months. However, many M. tuberculosis strains are resistant to one or more of the standard TB drugs, which complicates treatment greatly (Goodman \& Lipman, 2008). TB is a preventable and curable disease, prompt diagnosis and treatment remain the most effective means of controlling the disease. ( Roseanne,2012)

Nurses are responsible for educating and supporting patients at home, through follow-up clinics, and, where appropriate, running telephone help lines to 
detect medication side effects, and screening those who have been in close contact with infectious TB patients and perhaps running clinics for patients taking prophylactic TB treatment. Because of their frontline presence in all health-care facilities and their diversity of skills, nurses are the natural ally in the fight against TB. However, nurses in many countries lack adequate training in TB detection and management. (Alwan \& Hornby, 2002).

\section{Subjects and Methods: \\ Research design}

Cross sectional research design was used in this study.

Study sample:

After visiting the directorate of health and knowing the total number of TB patients (220) that were registered in the out patient clinics of chest hospitals in nine districts in Minia Governorate. By simple random sample three of them were chosen, then the researcher got their telephone numbers and their addresses from each health district, the total number of sample were $125 \mathrm{~TB}$ patients and 173 family caregivers. After that, the researcher contact with family in their home to complete the structured interview questionnaire sheet.

Inclusion criteria:

All adult TB patients and their family caregivers who are present during home visits, and willing to participate in the study, aging 18 years and above were included.

\section{Exclusion criteria:}

Children under 18 years, elderly above 60 years with severe health problems, patients in critical cases, severely ill and comatosed patients.

\section{Tools:-}

Two tools were used in this study

\section{Tool 1:}

Interview questionnaire sheet was constructed after reviewing the relevant literature to assess knowledge of TB patients and family caregivers about TB and it consists of three parts:

\section{Part 1:}

This part covered the following information sociodemographic characteristics of patients and their families which include (age, sex, address, occupation, income..etc) according to (Abd El-Tawab, 2004).

Part 2:

Which include environmental assessment sheet which was developed by the researcher, to assess home sanitation such as (Ventilation, presence of sewage sanitation and home facilities and crowdness by estimation of crowding index ....etc).

\section{Part 3:}

It includes questions to assess knowledge of TB patients and their family caregivers about tuberculosis disease such as (seriousness of TB, signs \& symptoms, modes of transmission, high risk group, dangerous of disease, investigations, treatment and prevention and source of information) These information were quoted from survey of knowledge, attitude and practice enhanced response to TB, Egypt (WHO, 2008).

\section{Tool 2:}

A rating scale used to assess attitude of patients' and their families about tuberculosis and their stigma in community. It was included (13) items for patients and their families. The response were on a three-point Likert scale ranging from (I don't know, agree and disagree).Items were scored $(0,1,2)$, respectively (WHO, 2008) in English form and translated into Arabic The validity of this tool was checked and revised by panel of seven experts from nursing and medical staff who reviewed the instrument for clarity, relevance, comprehensiveness, understanding and applicability. The reliability of the questionnaire was assessed using cronbachs alpha with an overall internal consistency of 0.75 .

\section{Methods:}

\section{I- Administrative design}

An official approval letter to conduct the study was obtained from the Dean of the Faculty of Nursing, Minia University to the Managers of the previous mentioned chest hospitals to facilitate the study; this letter included the title and the aim of the study.

The first contact between the researcher and the study participants was at the outpatient clinic. During this interview the personal characteristics of patients were obtained that include address and telephone numbers then the second interview were carried out with the study participants by visiting them at home.

\section{II- Ethical consideration}

The purpose of the study were explained for every TB patient and their family caregivers and the investigator promised them for confidentiality of information and that they have ethical right to agree or refuse participation in the study, an oral consent was taken from those who agreed to participate in the study.

\section{III- Pilot study}

A pilot study was done on $(10 \%)$ patients and their family caregivers from the total sample and excluded from the study sample. The aim of pilot study was to test the clarity of the tools and to estimate the required time to fill the questionnaire. Based on the result of this pilot study, some modifications in the tools were done. 


\section{IV- Data Collection}

Data were collected through interviewing patients and their families at their homes. The estimated time to complete each interview was 20-30 minutes, depending on the response of participants. Data collection was started from (February 2012 to June 2012), three days per week, and the average number of interviews was 4-5 per day.

\section{V- Statistical design and scoring system}

The collected data were tabulated and statistically analyzed to evaluate the difference between the groups under study as regards the various variables. The statistical analysis was done using SPSS program version 16, descriptive and inferential data were carried out including; frequency, percentage, chisquare and ANOVA test.

A scoring system was designed for the assessment of knowledge, 1 grade was given for each correct answer and zero was given for an incorrect answer and I don't know. The grade of each item summedup and then converted into a percent score. Poor knowledge: score less than 50\%, Fair knowledge: if score is $50-70 \%$ and Good knowledge: if score more than $70 \%$ (Solliman et al, 2012)

Regarding to total scoring of attitude scale, it consists of (13) questions. Each question is scored using a three point Likert scale. It was calculated by summing-up and then converted into a percent score. Patient and their families attitude were considered positive attitude if the score was more than $60 \%$ and negative attitude if the score less than $60 \%$. (Ibrahim et al, 2009)

\section{Results:}

Table (1) shows Sociodemographic characteristics of the studied sample. Around half (49.6\%) of patients aged ranged between 20-39 years, with mean age of $34.8 \pm 13.7$. while $47.4 \%$ of family caregivers were in age group 40-60 years with mean age 38.8 \pm 13.9 . Regarding sex, the study sample revealed that female patients are more than male $54.4 \%$ versus $45.6 \%$, while in the family caregivers male gender are more involved than female (52.6\% versus $47.4 \%$ ).

Regarding to education, it was found that less than half of patients and their family caregivers (43.2\% and $43.9 \%$ ) were illiterate, while only $4.8 \%$ and $2.9 \%$ of them had university education - Concerning residence, the majority $(80.8 \%)$ of the studied sample lived in rural, while $(19.2 \%)$ were from urban. As regard to social score, about two thirds $(61.6 \%)$ of patients were from middle social class, while $(16.8 \%)$ had high social class.

Table (2) shows the knowledge of patient and their families about mode of transmission of $\mathrm{TB}$, it was observed that more than half of both TB patients and their families ( $59.2 \%$ and $54.3 \%$ ) respectively did not know the mode of transmission of TB. While more than one third $(37.6 \%$ and $33.5 \%)$ respectively of studied sample stated that smoking is the mode of transmission of TB followed by air, when TB patient coughs or sneezes $(11.2 \%$ and $16.2 \%)$, respectively.

Table (3) illustrates the knowledge of patient and their families about high risk groups for TB. It was observed that $(57.6 \%$ and $62.4 \%)$ respectively of studied sample did not know the high risk group of TB. While $23.2 \%$ of patients stated that drug users is highest people risk to TB infection and $21.4 \%$ of family caregivers stated that alcoholics is high people risk to TB.

Table (4) shows the knowledge of TB patients and their families about sign \& symptoms of TB, it was observed that more than half $(58.4 \%)$ of the studied TB patients stated cough that last more than 3 weeks is the main sign \& symptoms of TB followed by shortness of breath, coughing blood, and fever without clear cause that last more than 7 days 56\%, $50.4 \%, 48 \%$ respectively. While it was observed that around half( $49.7 \%$ ) of family of studied sample stated that cough that last more than 3 weeks and coughing blood is the main signs \& symptoms of TB followed by Shortness of breath and fever without clear cause that last more than 7 days $46.2 \%, 24.8 \%$ respectively.

Table (5) shows the knowledge of TB patients and their families about different types of investigations of TB, it was observed that the majority $(88.8 \%$, $84.4 \%$ ) of studied sample stated that Chest $\mathrm{x}$-ray is the main type of investigation followed by sputum analysis $(78.4 \%, 57.8 \%)$ respectively.

Table (6) shows the knowledge of TB patients and their families about prevention of $\mathrm{TB}$, it was observed that nearly half of the studied sample (44.8\%) did not know preventive measure of TB. While more than one third of studied sample (38.4\%) state that good nutrition is the best mode of prevention of TB.

Table (7) illustrate the relation between knowledge of TB patients and their sociodemographic characteristics, as regard to age, it was observed that around three quarter $(73.8 \%)$ of patient had poor score in the age group between 40-60 years. While good score was observed in $17.7 \%$ their age from 20 39 years with no statistically significant difference $(P$ $=0.2$ ). As regard to patient education, more than three quarter $(79.6 \%)$ of illiterate had poor knowledge, while good knowledge was observed in $50 \%$ of university education with high statistically significant difference $(\mathrm{P}=0.0001)$. Also $(100 \%)$ of widowed patients had poor knowledge, versus to $(19.4 \%)$ of single had good knowledge with no statistically significant difference $(\mathrm{P}=0.264)$. 
Table (8) shows the relation between knowledge of family caregivers and sociodemographic characteristics, as regard to age it was observed that two third $(66.7 \%)$ had poor score of knowledge in age group less than 20 years, while $(11 \%)$ were good knowledge between age group 40-60- years. With statistically significant difference $(\mathrm{P}=.002)$. Regarding education, three quarter $(75 \%)$ of illiterate had poor score of knowledge, while $80 \%$ of university education had good score of knowledge. with high statistically significant difference ( $P$ $=0.0001$ ).

Table (9) shows the total knowledge score of the TB patients and their families about TB, it was observed that about two thirds $(64.8 \%)$ of patient had poor knowledge, $23.2 \%$ had fair knowledge and $12 \%$ had good knowledge, while more than half $(54.3 \%)$ of family had poor knowledge, $29.5 \%$ had fair knowledge and $16.2 \%$ had good knowledge

Table (10) shows that more than two third $(66.4 \%)$ of TB patients agree that he had ashamed from TB and $71.2 \%$ hide that he had infection. While the majority of patients agree that TB affect family responsibilities, work performance, breast feeding and lead to serious complications during pregnancy. (98.4\%. 97.6\%. 84,8\% and 82.4\%) respectively. Also $(50.4 \%)$ disagree that TB cause female infertility.

Table (11) shows that more than three quarter (76.9\%) of patients agree that they would be ashamed from $\mathrm{TB}$ and $78 \%$ hide that they had a patient with infection. While the majority of patients (95.4\%) agree that TB affect family responsibilities, work performance, breast feeding and lead to serious complications during pregnancy. (95.4\%, 92.5\%, $79.2 \%$ and $75.1 \%$ ) respectively Also $49.1 \%$ disagree that TB cause female infertility

Table (12) shows the total attitude score of the TB patients and their families about TB, it was observed that more than three quarter (77.6\%) of patients and ( $78 \%$ ) of family caregivers had negative attitude and $22.4 \%$ and $22 \%$ respectively of them had positive attitude.

Table (1) Sociodemographic characteristics of the TB patients and their family caregivers, Minia Governorate, 2013

\begin{tabular}{|c|c|c|c|c|}
\hline \multirow{2}{*}{ Variables } & \multicolumn{2}{|c|}{$\begin{array}{l}\text { Patients } \\
(\mathrm{n}=125)\end{array}$} & \multicolumn{2}{|c|}{$\begin{array}{l}\text { Families } \\
(n=173)\end{array}$} \\
\hline & No. & $\%$ & No. & $\%$ \\
\hline \multicolumn{5}{|l|}{ Age ( years ): } \\
\hline Less than 20 & 21 & 16.8 & 18 & 10.4 \\
\hline $20-39$ & 62 & 49.6 & 73 & 42.2 \\
\hline $40-60$ & 42 & 33.6 & 82 & 47.4 \\
\hline Mean \pm SD & \multicolumn{2}{|c|}{$34.8 \pm 13.7$} & \multicolumn{2}{|c|}{$38.8 \pm 13.9$} \\
\hline \multicolumn{5}{|l|}{ Sex: } \\
\hline Male & 57 & 45.6 & 91 & 52.6 \\
\hline Female & 68 & 54.4 & 82 & 47.4 \\
\hline \multicolumn{5}{|l|}{ Educational level: } \\
\hline Illiterate & 54 & 43.2 & 76 & 43.9 \\
\hline Read and write & 8 & 6.4 & 13 & 7.5 \\
\hline $\begin{array}{l}\text { Primary } \\
\text { education }\end{array}$ & 21 & 16.8 & 29 & 16.8 \\
\hline $\begin{array}{l}\text { Secondary } \\
\text { education }\end{array}$ & 36 & 28.8 & 50 & 28.9 \\
\hline $\begin{array}{l}\text { Universal } \\
\text { education }\end{array}$ & 6 & 4.8 & 5 & 2.9 \\
\hline \multicolumn{5}{|l|}{ Residence: } \\
\hline Rural & 101 & 80.8 & 140 & 80.9 \\
\hline Urban & 24 & 19.2 & 33 & 19.1 \\
\hline \multicolumn{5}{|l|}{ Social score: } \\
\hline Low & 27 & 21.6 & 37 & 21.4 \\
\hline Middle & 77 & 61.6 & 106 & 61.3 \\
\hline High & 21 & 16.8 & 30 & 17.3 \\
\hline
\end{tabular}

Table (2) Knowledge of TB patients and their family caregivers about mode of transmission of TB, Minia Governorate, 2013

\begin{tabular}{|l|c|c|c|c|}
\hline \multirow{2}{*}{\multicolumn{1}{|c|}{ Variables }} & \multicolumn{2}{c|}{$\begin{array}{c}\text { Patients } \\
\text { (n= 125) }\end{array}$} & \multicolumn{2}{c|}{$\begin{array}{c}\text { Families } \\
\text { (n= 173) }\end{array}$} \\
\cline { 2 - 5 } & No. & \% & No. & \% \\
\hline Don't know & 74 & 59.2 & 94 & 54.3 \\
\hline Smoking & 47 & 37.6 & 58 & 33.5 \\
\hline $\begin{array}{l}\text { Air, when TB patient } \\
\text { coughs or sneezes }\end{array}$ & 14 & 11.2 & 28 & 16.2 \\
\hline $\begin{array}{l}\text { Sharing Sheesha } \\
\text { pipes }\end{array}$ & 14 & 11.2 & 18 & 10.4 \\
\hline $\begin{array}{l}\text { Eating in same } \\
\text { dishes }\end{array}$ & 8 & 6.4 & 20 & 11.6 \\
\hline
\end{tabular}

There is more than one answer

Table (3) Knowledge of TB patients and their family caregivers about high risk groups of TB, Minia Governorate, 2013
Variables
Patients
Families 


\begin{tabular}{|l|c|c|c|c|}
\hline \multirow{2}{*}{} & \multicolumn{2}{|c|}{$(\mathbf{n = 1 2 5})$} & \multicolumn{2}{c|}{$(\mathbf{n = 1 7 3})$} \\
\cline { 2 - 5 } & No. & \% & No. & \% \\
\hline Don't know & 72 & 57.6 & 108 & 62.4 \\
\hline Alcoholics & 29 & 23.2 & 37 & 21.4 \\
\hline Drug users & 34 & 27.2 & 29 & 16.8 \\
\hline Anybody & 21 & 16.8 & 22 & 12.7 \\
\hline Homeless people & 4 & 3.2 & 10 & 5.8 \\
\hline $\begin{array}{l}\text { Close contacts with } \\
\text { infected patients }\end{array}$ & 7 & 5.6 & 10 & 5.8 \\
\hline Smokers & 6 & 4.8 & 4 & 2.3 \\
\hline
\end{tabular}

There is more than one answer

Table (4) Knowledge of TB patients and their family caregivers about signs \& symptoms of TB, Minia Governorate, 2013

\begin{tabular}{|l|c|c|c|c|}
\hline \multirow{2}{*}{\multicolumn{1}{|c|}{ Variables }} & \multicolumn{2}{c|}{$\begin{array}{c}\text { Patients } \\
\text { (n= 125) }\end{array}$} & \multicolumn{2}{c|}{$\begin{array}{c}\text { Families } \\
\text { (n= 173) }\end{array}$} \\
\cline { 2 - 5 } & No. & \% & No. & \% \\
\hline $\begin{array}{l}\text { Cough that last more } \\
\text { than 3 weeks }\end{array}$ & 73 & 58.4 & 86 & 49.7 \\
\hline Shortness of breath & 70 & 56.0 & 80 & 46.2 \\
\hline Coughing blood & 63 & 50.4 & 86 & 49.7 \\
\hline $\begin{array}{l}\text { Fever without clear } \\
\text { cause that last more } \\
\text { than 7 days }\end{array}$ & 60 & 48 & 43 & 24.8 \\
\hline Weakness & 34 & 27.2 & 28 & 16.2 \\
\hline
\end{tabular}

\begin{tabular}{|l|c|c|c|c|}
\hline \multirow{2}{*}{\multicolumn{1}{|c|}{ Variables }} & \multicolumn{2}{c|}{$\begin{array}{c}\text { Patients } \\
(\mathbf{n = 1 2 5})\end{array}$} & \multicolumn{2}{c|}{$\begin{array}{c}\text { Families } \\
(\mathbf{n = 1 7 3})\end{array}$} \\
\cline { 2 - 5 } & No. & \% & No. & \% \\
\hline Chest pain & 29 & 23.2 & 23 & 13.3 \\
\hline Weight loss & 25 & 20.0 & 32 & 18.5 \\
\hline $\begin{array}{l}\text { Pain in different site } \\
\text { of the body }\end{array}$ & 24 & 19.2 & 21 & 12.1 \\
\hline Severe headache & 7 & 5.6 & 11 & 6.4 \\
\hline
\end{tabular}

There is more than one answer

Table (5) Knowledge of TB patients and their family caregivers about diagnostic methods of TB, Minia Governorate, 2013

\begin{tabular}{|l|c|c|c|c|}
\hline \multirow{2}{*}{\multicolumn{1}{|c|}{ Variables }} & \multicolumn{2}{c|}{$\begin{array}{c}\text { Patients } \\
(\mathrm{n}=125)\end{array}$} & \multicolumn{2}{c|}{$\begin{array}{c}\text { Families } \\
(\mathrm{n}=173)\end{array}$} \\
\cline { 2 - 5 } & No. & $\%$ & No. & $\%$ \\
\hline Chest x-ray & 111 & $\mathbf{8 8 . 8}$ & 146 & 84.4 \\
\hline Sputum analysis & 98 & 78.4 & 100 & 57.8 \\
\hline Blood analysis & 30 & 24.0 & 20 & 11.6 \\
\hline CT scan \& MRI & 19 & 15.2 & 13 & 7.5 \\
\hline Urine analysis & 6 & 4.8 & 7 & 4.0 \\
\hline TB skin test & 5 & 4.0 & 2 & 1.2 \\
\hline ECG & 3 & 2.4 & 1 & 0.6 \\
\hline Ultrasound & 3 & 2.4 & 5 & 2.9 \\
\hline Sputum culture & 1 & 0.8 & 4 & 2.3 \\
\hline
\end{tabular}

There is more than one answer

Table (6) Knowledge of TB patients and their family caregivers about preventive measures of TB, Minia Governorate, 2013

\begin{tabular}{|l|c|c|c|c|}
\hline \multicolumn{1}{c|}{ Variables } & \multicolumn{2}{c|}{$\begin{array}{c}\text { Patients } \\
(\mathbf{n = 1 2 5})\end{array}$} & \multicolumn{2}{c|}{$\begin{array}{c}\text { Families } \\
(\mathbf{n = 1 7 3})\end{array}$} \\
\cline { 2 - 5 } & No. & \% & No. & \% \\
\hline Don't know & 56 & 44.8 & 78 & 45.1 \\
\hline Good nutrition & 48 & 38.4 & 62 & 35.8 \\
\hline Stop smoking & 31 & 24.8 & 27 & 15.6 \\
\hline Avoid sharing TB patients utensils & 19 & 15.2 & 45 & 26.0 \\
\hline Taking complete treatment & 12 & 9.6 & 9 & 5.2 \\
\hline Covering mouth and nose when coughing or sneezing & 5 & 4.0 & 4 & 2.3 \\
\hline Good ventilation & 3 & 2.4 & 3 & 1.7 \\
\hline Washing hands after touching items in public places & 4 & 3.2 & 7 & 4.1 \\
\hline
\end{tabular}

There is more than one answer 
Table (7) Relationship between sociodemographic characteristics of TB patients and their knowledge about the disease, Minia Governorate, 2013

\begin{tabular}{|c|c|c|c|c|c|c|c|}
\hline \multirow{3}{*}{ Variables } & \multicolumn{6}{|c|}{ Knowledge } & \multirow{3}{*}{$\begin{array}{l}\text { P-value } \\
\left(\mathrm{X}^{2}\right)\end{array}$} \\
\hline & \multicolumn{2}{|c|}{$\begin{array}{c}\text { Poor } \\
(n=81)\end{array}$} & \multicolumn{2}{|c|}{$\begin{array}{c}\text { Satisfactory } \\
(n=29)\end{array}$} & \multicolumn{2}{|c|}{$\begin{array}{c}\text { Good } \\
(n=15)\end{array}$} & \\
\hline & No. & $\%$ & No. & $\%$ & No. & $\%$ & \\
\hline $\begin{array}{l}\text { Age: } \\
\text { Less than } 20 \\
20-39 \\
40-60 \\
\end{array}$ & $\begin{array}{l}13 \\
37 \\
31 \\
\end{array}$ & $\begin{array}{l}61.9 \% \\
59.7 \% \\
73.8 \%\end{array}$ & $\begin{array}{c}7 \\
14 \\
8 \\
\end{array}$ & $\begin{array}{l}33.3 \% \\
22.6 \% \\
19.1 \% \\
\end{array}$ & $\begin{array}{c}1 \\
11 \\
3 \\
\end{array}$ & $\begin{array}{c}4.8 \% \\
17.7 \% \\
7.1 \%\end{array}$ & $\begin{array}{c}0.2 \\
(5.5)\end{array}$ \\
\hline $\begin{array}{l}\text { Sex: } \\
\quad \text { Male } \\
\text { Female } \\
\end{array}$ & $\begin{array}{l}27 \\
54 \\
\end{array}$ & $\begin{array}{l}47.4 \% \\
79.4 \% \\
\end{array}$ & $\begin{array}{l}19 \\
10 \\
\end{array}$ & $\begin{array}{l}33.3 \% \\
14.7 \% \\
\end{array}$ & $\begin{array}{c}11 \\
4 \\
\end{array}$ & $\begin{array}{c}19.3 \% \\
5.9 \%\end{array}$ & $\begin{array}{c}0.001 * \\
(14.202) \\
\end{array}$ \\
\hline $\begin{array}{l}\text { Education level: } \\
\text { Illiterate } \\
\text { Read and write } \\
\text { Primary education } \\
\text { Secondary education } \\
\text { Universal education }\end{array}$ & $\begin{array}{c}43 \\
5 \\
15 \\
17 \\
1\end{array}$ & $\begin{array}{l}79.6 \% \\
62.5 \% \\
71.4 \% \\
47.2 \% \\
16.7 \%\end{array}$ & $\begin{array}{l}10 \\
2 \\
6 \\
9 \\
2\end{array}$ & $\begin{array}{c}18.5 \% \\
25 \% \\
28.6 \% \\
25 \% \\
33.3 \%\end{array}$ & $\begin{array}{c}1 \\
1 \\
0 \\
10 \\
3\end{array}$ & $\begin{array}{c}1.9 \% \\
12.5 \% \\
.0 \% \\
27.8 \% \\
50 \%\end{array}$ & $\begin{array}{l}0.0001 * \\
(28.786)\end{array}$ \\
\hline $\begin{array}{l}\text { Marital status: } \\
\text { Single } \\
\text { Married } \\
\text { Widowed } \\
\end{array}$ & $\begin{array}{c}18 \\
57 \\
6 \\
\end{array}$ & $\begin{array}{c}58.1 \% \\
64.8 \% \\
100 \% \\
\end{array}$ & $\begin{array}{c}7 \\
22 \\
0 \\
\end{array}$ & $\begin{array}{c}22.6 \% \\
25 \% \\
.0 \% \\
\end{array}$ & $\begin{array}{r}6 \\
9 \\
0 \\
\end{array}$ & $\begin{array}{c}19.4 \% \\
10.2 \% \\
.0 \% \\
\end{array}$ & $\begin{array}{c}.264 \\
(5.232)\end{array}$ \\
\hline $\begin{array}{l}\text { Occupation: } \\
\text { Not work } \\
\text { Manual Worker } \\
\text { Professional } \\
\text { Employee } \\
\end{array}$ & $\begin{array}{c}57 \\
16 \\
7 \\
1 \\
\end{array}$ & $\begin{array}{c}76 \% \\
64 \% \\
46.7 \% \\
10 \% \\
\end{array}$ & $\begin{array}{l}13 \\
7 \\
5 \\
4 \\
\end{array}$ & $\begin{array}{c}17.3 \% \\
28 \% \\
33.3 \% \\
40 \% \\
\end{array}$ & $\begin{array}{l}5 \\
2 \\
3 \\
5\end{array}$ & $\begin{array}{c}6.7 \% \\
8 \% \\
20.0 \% \\
50 \% \\
\end{array}$ & $\begin{array}{l}0.0001 * \\
(25.036)\end{array}$ \\
\hline $\begin{array}{l}\text { Social score: } \\
\text { Low } \\
\text { Middle } \\
\text { High }\end{array}$ & $\begin{array}{l}21 \\
50 \\
10\end{array}$ & $\begin{array}{l}77.8 \% \\
64.9 \% \\
47.6 \%\end{array}$ & $\begin{array}{c}5 \\
16 \\
8\end{array}$ & $\begin{array}{l}18.5 \% \\
20.8 \% \\
38.1 \%\end{array}$ & $\begin{array}{c}1 \\
11 \\
3\end{array}$ & $\begin{array}{l}3.7 \% \\
14.3 \% \\
14.3 \%\end{array}$ & $\begin{array}{c}0.192 \\
(6.092)\end{array}$ \\
\hline $\begin{array}{c}\text { Residence: } \\
\text { Rural } \\
\text { Urban }\end{array}$ & $\begin{array}{l}68 \\
13 \\
\end{array}$ & $\begin{array}{l}67.3 \% \\
54.2 \%\end{array}$ & $\begin{array}{c}21 \\
8 \\
\end{array}$ & $\begin{array}{l}20.8 \% \\
33.3 \%\end{array}$ & $\begin{array}{c}12 \\
3\end{array}$ & $\begin{array}{l}11.9 \% \\
12.5 \%\end{array}$ & $\begin{array}{c}0.3 \\
(1.83)\end{array}$ \\
\hline
\end{tabular}

$*$ Statistical significant difference $(\mathrm{P}<0.05)$

Table (8) Relationship between sociodemographic characteristics of TB patients family caregivers and their knowledge, Minia Governorate, 2013

\begin{tabular}{|c|c|c|c|c|c|c|c|}
\hline \multirow{3}{*}{ Variables } & \multicolumn{6}{|c|}{ Knowledge } & \multirow{3}{*}{$\begin{array}{c}\text { P-value } \\
\left(\mathrm{X}^{2}\right)\end{array}$} \\
\hline & \multicolumn{2}{|c|}{$\begin{array}{c}\text { Poor } \\
(n=94)\end{array}$} & \multicolumn{2}{|c|}{$\begin{array}{c}\text { Satisfactory } \\
(n=51)\end{array}$} & \multicolumn{2}{|c|}{$\begin{array}{c}\text { Good } \\
(n=28)\end{array}$} & \\
\hline & No. & $\%$ & No. & $\%$ & No. & $\%$ & \\
\hline $\begin{array}{l}\text { Age: } \\
\text { Less than } 20 \\
20-39 \\
40-60\end{array}$ & $\begin{array}{l}12 \\
30 \\
52\end{array}$ & $\begin{array}{l}66.7 \% \\
41.1 \% \\
63.4 \%\end{array}$ & $\begin{array}{c}2 \\
28 \\
21\end{array}$ & $\begin{array}{l}11.1 \% \\
38.4 \% \\
25.6 \%\end{array}$ & $\begin{array}{c}4 \\
15 \\
9\end{array}$ & $\begin{array}{c}22.2 \% \\
20.5 \% \\
11 \%\end{array}$ & $\begin{array}{c}0.02 * \\
(11.16)\end{array}$ \\
\hline $\begin{array}{l}\text { Sex: } \\
\text { Male } \\
\text { Female }\end{array}$ & $\begin{array}{l}46 \\
48\end{array}$ & $\begin{array}{l}50.5 \% \\
58.5 \%\end{array}$ & $\begin{array}{l}26 \\
25\end{array}$ & $\begin{array}{l}28.6 \% \\
30.5 \%\end{array}$ & $\begin{array}{c}19 \\
9\end{array}$ & $\begin{array}{c}20.9 \% \\
11 \%\end{array}$ & $\begin{array}{c}0.2 \\
(3.17)\end{array}$ \\
\hline
\end{tabular}




\begin{tabular}{|c|c|c|c|c|c|c|c|}
\hline \multirow{3}{*}{ Variables } & \multicolumn{6}{|c|}{ Knowledge } & \multirow{3}{*}{$\begin{array}{c}\text { P-value } \\
\left(X^{2}\right)\end{array}$} \\
\hline & \multicolumn{2}{|c|}{$\begin{array}{c}\text { Poor } \\
(n=94)\end{array}$} & \multicolumn{2}{|c|}{$\begin{array}{c}\text { Satisfactory } \\
(n=51)\end{array}$} & \multicolumn{2}{|c|}{$\begin{array}{c}\text { Good } \\
(n=28)\end{array}$} & \\
\hline & No. & $\%$ & No. & $\%$ & No. & $\%$ & \\
\hline $\begin{array}{l}\text { Education level: } \\
\text { Illiterate } \\
\text { Read and write } \\
\text { Primary education } \\
\text { Secondary education } \\
\text { Universal education }\end{array}$ & $\begin{array}{c}57 \\
5 \\
16 \\
16 \\
0\end{array}$ & $\begin{array}{c}75 \% \\
38.5 \% \\
55.2 \% \\
32 \% \\
0.0 \%\end{array}$ & $\begin{array}{c}16 \\
6 \\
10 \\
18 \\
1\end{array}$ & $\begin{array}{l}21.1 \% \\
46.2 \% \\
34.5 \% \\
36 \% \\
20 \%\end{array}$ & $\begin{array}{c}3 \\
2 \\
3 \\
16 \\
4\end{array}$ & $\begin{array}{c}3.9 \% \\
15.4 \% \\
10.3 \% \\
32 \% \\
80 \%\end{array}$ & $\begin{array}{c}0.0001 * \\
(46.02)\end{array}$ \\
\hline $\begin{array}{l}\text { Marital status: } \\
\text { Single } \\
\text { Married } \\
\text { Widowed } \\
\end{array}$ & $\begin{array}{c}13 \\
77 \\
4\end{array}$ & $\begin{array}{c}43.3 \% \\
55.4 \% \\
100 \%\end{array}$ & $\begin{array}{c}11 \\
40 \\
0\end{array}$ & $\begin{array}{c}36.7 \% \\
28.8 \% \\
0.0 \%\end{array}$ & $\begin{array}{c}6 \\
22 \\
0\end{array}$ & $\begin{array}{l}20 \% \\
5.8 \% \\
0.0 \%\end{array}$ & $\begin{array}{c}0.2 \\
(4.88)\end{array}$ \\
\hline $\begin{array}{l}\text { Occupation: } \\
\text { Not work } \\
\text { Manual Worker } \\
\text { Professional } \\
\text { Employee } \\
\end{array}$ & $\begin{array}{c}54 \\
25 \\
11 \\
4 \\
\end{array}$ & $\begin{array}{c}60 \% \\
54.4 \% \\
55 \% \\
23.5 \% \\
\end{array}$ & $\begin{array}{c}25 \\
18 \\
5 \\
3 \\
\end{array}$ & $\begin{array}{c}27.8 \% \\
39.1 \% \\
25 \% \\
17.7 \% \\
\end{array}$ & $\begin{array}{c}11 \\
3 \\
4 \\
10\end{array}$ & $\begin{array}{l}12.2 \% \\
6.5 \% \\
20 \% \\
8.8 \% \\
\end{array}$ & $\begin{array}{c}0.0001 * \\
(28.79)\end{array}$ \\
\hline $\begin{array}{l}\text { Degree of kinship: } \\
\text { Father / Mother } \\
\text { Brother / Sister } \\
\text { Son / Daughter } \\
\text { Husband / Wife }\end{array}$ & $\begin{array}{c}28 \\
8 \\
16 \\
42\end{array}$ & $\begin{array}{c}58.3 \% \\
50 \% \\
45.7 \% \\
56.8 \%\end{array}$ & $\begin{array}{c}13 \\
6 \\
11 \\
21\end{array}$ & $\begin{array}{l}27.1 \% \\
37.5 \% \\
31.4 \% \\
28.4 \%\end{array}$ & $\begin{array}{c}7 \\
2 \\
8 \\
11\end{array}$ & $\begin{array}{l}14.6 \% \\
12.5 \% \\
22.9 \% \\
14.9 \%\end{array}$ & $\begin{array}{c}0.5 \\
(6.8)\end{array}$ \\
\hline
\end{tabular}

$*$ Statistical significant difference $(\mathrm{P}<0.05)$

Table (9) Total knowledge score of the TB patients and their family caregivers, Minia Governorate about TB, 2013

\begin{tabular}{|l|c|c|c|c|}
\hline \multirow{2}{*}{ Total knowledge } & \multicolumn{2}{|c|}{ Study participants } & \multirow{2}{*}{$\mathbf{X}^{\mathbf{2}}$} & \multirow{2}{*}{ P-value } \\
\cline { 2 - 3 } & Patients & Families & & \\
\hline Poor & $81(64.8 \%)$ & $94(54.3 \%)$ & & \multirow{2}{*}{3.2} \\
\hline Fair & $29(23.2 \%)$ & $51(29.5 \%)$ & \\
\cline { 1 - 3 } Good & $15(12 \%)$ & $28(16.2 \%)$ & & \\
\hline Total & $125(100 \%)$ & $173(100 \%)$ & & \\
\hline
\end{tabular}

Table (10) Distribution of TB patient's attitude about tuberculosis, Minia Governorate, 2013

\begin{tabular}{|l|c|c|c|c|c|c|}
\hline \multicolumn{1}{|c|}{ Variables } & \multicolumn{2}{c|}{ Agree } & \multicolumn{2}{c|}{ Disagree } & \multicolumn{2}{c|}{ Don't know } \\
\cline { 2 - 7 } & No. & $\mathbf{\%}$ & No. & \% & No. & \% \\
\hline $\begin{array}{l}\text { 1- In case you had TB, would you be ashamed for } \\
\text { having TB? }\end{array}$ & 83 & 66.4 & 27 & 21.6 & 15 & 12 \\
\hline 2- Would you want to hide that you have TB? & 89 & 71.2 & 25 & 20 & 11 & 8.8 \\
\hline $\begin{array}{l}\text { 3- TB affect relationship with others } \\
\text { 4- Would you prefer to live isolated in case you had } \\
\text { TB? }\end{array}$ & 83 & 66.4 & 22 & 17.6 & 20 & 16 \\
\hline 5- TB affect your work performance & 40 & 35 & 28 & 30 & 24 \\
\hline 6- TB affect your marital relationship & 122 & 97.6 & 2 & 1.6 & 1 & .8 \\
\hline 7- TB affect your family responsibilities & 123 & 67.2 & 14 & 11.2 & 27 & 21.6 \\
\hline $\begin{array}{l}\text { 8- There are less chances of getting married due to } \\
\text { TB diagnosis }\end{array}$ & 60 & 48 & 10 & 8 & 55 & 44 \\
\hline 9- TB can affect female fertility & 14 & 11.2 & 63 & 50.4 & 48 & 38.4 \\
\hline 10- TB leads to serious complication during & 103 & 82.4 & 3 & 2.4 & 19 & 15.2 \\
\hline
\end{tabular}




\begin{tabular}{|c|c|c|c|c|c|c|}
\hline \multirow{2}{*}{ Variables } & \multicolumn{2}{c|}{ Agree } & \multicolumn{2}{c|}{ Disagree } & \multicolumn{2}{c|}{ Don't know } \\
\cline { 2 - 7 } & No. & $\mathbf{\%}$ & No. & $\mathbf{\%}$ & No. & $\%$ \\
\hline pregnancy & & & & & & \\
\hline 11- TB can affect breast feeding & 106 & 84.8 & 1 & .8 & 18 & 14.4 \\
\hline $\begin{array}{l}\text { 12- TB can affect pregnancy outcome } \\
\begin{array}{l}\text { 13- Females get difficulty in deciding to be treated } \\
\text { for TB }\end{array}\end{array}$ & 59 & 49.6 & 6 & 4.8 & 57 & 45.6 \\
\hline
\end{tabular}

Table (11) Attitude of TB patient's family caregivers about tuberculosis, Minia Governorate, 2013

\begin{tabular}{|l|c|c|c|c|c|c|}
\hline \multicolumn{1}{|c|}{ Variables } & \multicolumn{2}{c|}{ Agree } & \multicolumn{2}{c|}{ Disagree } & \multicolumn{2}{c|}{ Don't know } \\
\cline { 2 - 6 } & No. & $\%$ & No. & \% & No. & \% \\
\hline $\begin{array}{l}\text { 1- In case of patient had TB, would you be } \\
\text { ashamed for having TB? }\end{array}$ & 133 & 76.9 & 22 & 12.7 & 18 & 10.4 \\
\hline $\begin{array}{l}\text { 2- Would you want to hide that the patient had TB } \\
\text { from the family? }\end{array}$ & 135 & 78 & 26 & 15.1 & 12 & 6.9 \\
\hline 3- TB affect patient relationship with others & 133 & 76.9 & 18 & 10.4 & 22 & 12.7 \\
\hline $\begin{array}{l}\text { 4- Would you prefer the patient live isolated in } \\
\text { case you had TB? }\end{array}$ & 77 & 44.5 & 53 & 30.6 & 43 & 24.9 \\
\hline 5- TB affect your work performance & 160 & 92.5 & 4 & 2.3 & 9 & 5.2 \\
\hline 6- TB affect your marital relationship & 125 & 72.3 & 20 & 11.5 & 28 & 16.2 \\
\hline 7- TB affect your family responsibilities & 165 & 95.4 & 1 & .6 & 7 & 4 \\
\hline $\begin{array}{l}\text { 8- There are less chances of getting married due to } \\
\text { TB diagnosis }\end{array}$ & 81 & 46.8 & 11 & 6.4 & 81 & 46.8 \\
\hline 9- TB can affect female fertility & 19 & 11 & 85 & 49.1 & 69 & 39.9 \\
\hline $\begin{array}{l}\text { 10- TB leads to serious complication during } \\
\text { pregnancy }\end{array}$ & 130 & 75.1 & 6 & 3.5 & 37 & 21.4 \\
\hline 11- TB can affect breast feeding & 137 & 79.2 & 5 & 2.9 & 31 & 17.9 \\
\hline 12- TB can affect pregnancy outcome & 72 & 41.6 & 19 & 11 & 82 & 47.4 \\
\hline $\begin{array}{l}\text { 13- Females get difficulty in deciding to be treated } \\
\text { for TB }\end{array}$ & 66 & 38.2 & 16 & 9.2 & 91 & 52.6 \\
\hline
\end{tabular}

Table (12) Total attitude score of the TB patients and their family caregivers about TB, Minia Governorate, 2013

\begin{tabular}{|l|c|c|c|c|}
\hline \multirow{2}{*}{ Total attitude } & \multicolumn{2}{|c|}{ Studied groups } & \multirow{2}{*}{$\mathbf{X}^{2}$} & \multirow{2}{*}{ P value } \\
\cline { 2 - 3 } & Patients & Families & & \multirow{2}{*}{0.9} \\
\hline Negative & $97(77.6 \%)$ & $135(78 \%)$ & & 1 \\
\hline Positive & $28(22.4 \%)$ & $38(22 \%)$ & & \\
\hline Total & $125(100 \%)$ & $173(100)$ & & \\
\hline
\end{tabular}

\section{Discussion:}

Tuberculosis is one of the most serious health problems globally. About one third of the global population is estimated to be infected with mycobacterium tuberculosis which kills an estimated two million of the approximately eight million people who develop the disease each year (Nkulu et al, 2010). It is spread through the air when people who have an active TB infection cough, sneeze, or otherwise transmit their saliva through the air. (Kethireddy, 2010)
In agreement with (Mohamed et al, 2007) showed that around half of patients were in age group 20$39 y e a r s$ old. But in family caregivers the present study showed $(47.4 \%)$ of family caregivers ranged between 40-60 years. Contradicted with (Ige \& lasebikan, 2011) who found more than two fifth (42\%) of age group 40-54. Regarding sex the present study show that about more than two fifth $(45.6 \%)$ of patients were male and more than half $(54.4 \%)$ were female. This contradicted with (Hashim et al., 2003) in Iraq reported that about $(66.6 \%)$ males and (33.4\%) females. And consistent with (Hoa et al, 2009) in a rural community in Vietnam reported that 
the majority $(75 \%)$ were female and $(25 \%)$ were male.

Regarding education level the present study showed that about more than two fifth $(43.2 \%)$ illiterate, $(6.4 \%)$ read and write, $(16.8 \%)$ primary education, $(28.8 \%)$ secondary education and (4.8\%) university education. This consistent with (Abd El-Hameed et al, 2012) at Benha University reported that (29\%) illiterate, $(21 \%)$ read and write $(13 \%)$ primary education, (30\%) secondary education and (7\%) university education.

Regarding mode of transmission the present study show that more than one third of patients and their family caregivers stated that smoking is the most common mode of transmission of $\mathrm{TB}$, this result contradicted with (Kar \& Logaraj, 2010; Legesse et al, 2010) were found air when cough or sneeze is the most common mode of the transmission of TB.

In agreement with (Mushtaq et al, 2010) the present study illustrated that drug users is the first high risk group of TB disease stated by patient, but in family caregivers stated that alcoholics is the first high risk group of TB, this contradicted with (Gilpin et al, 2011) who found the poor is the first high risk group of TB disease.

In agreement with (Yadav et al., 2006; Agboatwalla et al, 2003) the present study showed that more than half of patients and their family caregivers stated that cough last more than 3 weeks was the commonest symptom of TB.

Regarding diagnosis the present study showed that majority of patients stated that chest $\mathrm{X}$-ray is the most common diagnostic method of $\mathrm{TB}$, this consistent with (Bacay-Domingo, 2009) and in family caregivers stated that chest $\mathrm{x}$-ray is the most common diagnostic method of TB, this contradicted with (Baldwin et al, 2010) who found that sputum sample is the most common diagnostic method of TB.

Regarding duration of treatment the present study showed that the majority $(97.6 \%)$ of patients and their families knew the correct duration for the treatment of tuberculosis. this contradicted with (Koay, 2004. Needhan et al, 2004) in Kudat District, Sabah reported that about $33 \%$ of the respondents knew the correct duration for the treatment of tuberculosis.

The current study shows that more than one third of patients were mentioned that good nutrition is the most common prevention of $\mathrm{TB}$, this contradicted with (Anjum et al,2009) who reported that use of medicines is the most common prevention of $\mathrm{TB}$, and more than one third of family caregivers were mentioned that good nutrition is the most common prevention of TB, this contradicted with (Mfinanga et al, 2003) who reported that covering the mouth and nose while coughing and sneezing is the most common prevention of TB.
Regarding source of information the present study showed that majority ( $96.8 \%$ ) considered doctor is the most source of information. This contradicted with (Rahman et al, 2012., Jaramillo, 2001) in Bangladesh reported that about $46.8 \%$ stated that they get information about TB from television (46.8\%),

In agreement with (Aryal et al, 2012; Rintiswati et al, 2009) the present study showed that the majority of patients and their family caregivers wanted to hide disease from others. The present study shows that less than half $(44.5 \%)$ of patients and their family caregivers were agreed to isolate the TB patients from the family. This contradicted with (Kelly, 2010; Storla et al, 2008).

In conclusion, the present study concluded that the majority of patient age groups were (20-39) years. The majority of patients and their families had poor knowledge and negative attitude toward TB. So the present study recommended that health education for the public through campaigns about TB (Causes, mode of transmission, Sign and symptoms, complication, and prevention) through announcement on radio, TV and newspapers, magazines and pamphlets, posters in public places.

\section{References:}

1. Abd ElHameed, H.S., Aly, H.A., \& Mahdy, A.Y.,(2012): Effect of Counseling on Self-Care Management among Adult Patients with Pulmonary Tuberculosis, Life Science Journal;9(1):956-964.

2. Abdel-twab (2004): socioeconomic scale, faculty of education, Assiut University.

3. Agboatwalla, M., Kazi, G., Shah, S., \& Saeed, S., (2003): Gender Perspectives in Knowledge, Attitudes and Practices Concerning Tuberculosis in Pakistan's Sindh Province, PP; 4-91.

4. Alwan, A., \& Hornby, P., (2002): The implications of health sector reform for health resources development. Bull World Health Organ; 80:56-60.

5. Anjum, A., Daud, S., \& Mukhtar, F., (2009): Tuberculosis awareness about spread and control; professional at Ghurki Trust Hospital, Lahore Med J; 16(1):61-66.

6. Aryal, S., Badhu, A., Pandey, S., Bhandari, A., Khatiwoda, P., Khatiwada P., \& Giri, A.,(2012) : Stigma Related to Tuberculosis Among Patients Attending DOTS Clinics of Dharan Municipality, Kathmandu university medical journal;.10 ( 1 ) 37:48-52.

7. Bacay-Domingo, M., (2009): A descriptive study of the Knowledge, attitude and practice on tuberculosis among treatment partners of 
pediatric patients in Tarlac city, PIDSP Journal; 10(1):1-7.

8. Baldwin, M., Yori, P., Ford, C., Moore, D., Gilman, R.,

\& Vidal, C., (2010): Tuberculosis and nutrition: disease perceptions and health seeking behavior of household contacts in the Peruvian Amazon. Int J Tuberc Lung Dis; 8(12):1484-91.

9. Centers for Disease Control and Prevention (CDC), (2009b): Updated guidelines for the use of nucleic acid amplification tests in the diagnosis of tuberculosis. MMWR Morb Mortal Wkly Rep; 58(1):7-10.

10. Goodman, A., \& Lipman, M., (2008): "Tuberculosis," Clinical Med. 8:531-534.

11. Hashim, D.S., Al Kubaisy, W., \& Al Dulayme, A., (2003): Knowledge, attitudes and practices survey among health care workers and tuberculosis patients in Iraq, Eastern Mediterranean Health Journal, Vol. 9, No. 4,pp;718-731.

12. Hoa, N.P, Thorson, A.E, Long, N.H \& Diwan, V.K., (2003): Knowledge of tuberculosis and associated health seeking behaviour among rural Vietnamese adults with a cough for at least three weeks.scandinavian journal of public health; 31(1) Suppl. 62: 59-65.

13. Ibrahim, H., Ahmed, A., El-Aziz, M., \& Hussien, A., (2009): Nutritional and exercise habits for prevention of osteoporosis among female adolescents in Assiut City secondary school,the new egyptian journal of medicine vol:41;No:4 , $1^{\text {st }}$ October 2009 ,Pp346-356.

14. Ige, O., \& Lasebikan, V.,(2011): Prevalence of depression in tuberculosis patients in comparison with non-tuberculosis family contacts visiting the DOTS clinic in a Nigerian tertiary care hospital and its correlation with disease pattern, Ment Health Fam Med; 8(4): 235-241.

15. Jaramillo, E., (2001): The impact of mediabased health education on tuberculosis diagnosis in Cali, Colombia. Health Policy Plan; 16:68-73.

16. Kar, M., \& Logaraj, M., (2010): Awareness, attitude and treatment seeking behavior regarding Tuberculosis in a rural area of Tamil Nadu. Indian J Tuberc; 57: 226 - 229.

17. Kelly, P., (2010): Isolation and stigma: the experience of patients with active tuberculosis in India. J Community Health Nurs, 16(4):233-41.

18. Kethireddy, S., (2010): Tuberculosis-A review of clinical features, differential diagnosis treatment available. 2 (2) : 206-244.

19. Koay,T.K.,(2004): Knowledge and Attitudes Towards Tuberculosis Among the People Living in Kudat District, Sabah, Med J Malaysia Vol 59 No 4:502-511.

20. Konstantinos, A., (2010): "Testing for tuberculosis". Australian Prescriber; 33:12-18. http://www.australianprescriber.com/magazine/3 3/1/12/18/

21. Kunimoto, D., Sutherland, K., \& Wooldrage, K., (2004): Transmission characteristics of tuberculosis in the foreign-born and the Canadian-born populations of Alberta, Canada. Int J Tuberc Lung Dis; 8:1213-20.

22. kyle, T., \& Carman, S., (2013):Essential of pediatric nursing,chapter 18 , nursing care of the child with a respiratory disorder, lippincott Williams \& wilkins, $2^{\text {nd }}$ ed;615-616.

23. Legesse, M., Ameni, G., Mamo, G., Medhin, G., Shawel, D., Bjune, G., \& Abebe, F., (2010): Knowledge and perception of pulmonary tuberculosis in pastoral communities in the middle and Lower Awash Valley of Afar region, Ethiopia. BMC Public Health, 10:187.

24. Mfinanga, S., Morkve, O., Kazwala, R., Cleaveland, S., Sharp, J., Shirima, G., \& Nilsen, R., (2003): Tribal differences in perception of tuberculosis: a possible role in tuberculosis control in Arusha, Tanzania. Int $\mathbf{J}$ Tuberc Lung Dis, 7:933-41.

25. Mohamed, A., Yousif, M., Ottoa, P., \& Bayoumi, A.,(2007): Knowledge of Tuberculosis: A Survey among Tuberculosis Patients in Omdurman, Sudan, Sudanese journal of public health;2(1);21-28.

26. Mushtaq, M., Majrooh, M., \& Ahmad, W., (2010): Knowledge, attitudes and practices regarding tuberculosis in two districts of Punjab, Pakistan. Int J Tuberc Lung Dis, 14(3):303-10.

27. Needhan, D.M, Bowman, D., Foster, S.D., Godfrey- Fausset, P.,(2004): Patient care seeking barriers and tuberculosis programme reform: a qualitative study Health Policy; 67(1):93-106.

28. Nkulu, F., Hurtig,A., Ahlm, C., \& Krantz, I., (2010): Screening migrants for tuberculosisamissed opportunity for improving knowledge and attitude in high-risk groups: Across-sectional study of Swedish language students in Umea, Sweden, BMC Public health 10:349.

29. Rahman, A., Saria,T., \& Hoque, F.M.,(2012): Patient's Knowledge and Attitude towards Tuberculosis in an Urban Setting, Pulm Med. 2012;32-45.

30. Rintiswati, N., Mahendradhata, Y., \& Suharna, (2009): Journeys to tuberculosis treatment: a qualitative study of patients, families and communities in Jogjakarta, Indonesia. BMC Public Health; 9: 158. 
31. Roseanne E., (2012): Exploration of the knowledge about and attitudes about tuberculosis among non-TB infected attendees at a Cape Town community clinic, pp: 1-131.

32. Sillah, D., Hill, P., Brookes, R., Dondor, S., Lugos, M., Fox, A., Howie, S., Fielding, K., Jallow, A., Lienhart, C., Corrah, T., Adegbola, R., \& Mcadams, K., (2007): Screening for tuberculosis among 2381 households contacts of sputum smear positive cases in the Gambia. Transactions of the Royal Society of Tropical Medicine and Hygiene, 101: 594-601.

33. Solliman, M., Hassali, M., Al-Haddad, M., Hadida, M., Saleem, F., Atif, M., \& Aljadhey, H., (2012): Assessment of knowledge toward tuberculosis among general population in North East Libya, Journal of applied pharmaceutical science 2(4); 24-30.

34. Storla, D., Yimer, S., \& Bjune, G., (2008): A systematic review of delay in the diagnosis and treatment of tuberculosis. BMC Public Health; $14: 15$.

35. Wanyeki, I., Olson, S., \& Brassard, P., (2006): Dwellings, crowding, and tuberculosis in Montreal. Soc Sci Med: 63:501-11.

36. World Health Organization, (2008): KAP Survey of Knowledge, Attitudes and Practices Enhanced Response to TB ACSM, Egypt, pp; 136.

37. Yadav, S., Mathur, M., \& Dixit, A., (2006): Knowledge and attitude towards tuberculosis among sandstone quarry workers in desert parts of rajasthan, Indian J Tuberc; 53:187-195. 